\title{
LHCb Early Career Initiatives
}

\section{Martha Hilton, on behalf of the LHCb collaboration ${ }^{a, *}$}

${ }^{a}$ University of Manchester,

$U K$

E-mail: martha.hilton@cern.ch

Early career researchers in particle physics face a unique set of challenges. Newcomers to any experiment face steep learning curves, particularly with the familiarisation of software and internal standard procedure. The junior rank typical of early career researchers can also mean less agency or influence within a larger collaboration, and job insecurity brings further additional challenges, such as migration for work and a resulting lack of a consistent support network. These proceedings will discuss existing early career initiatives at $\mathrm{LHCb}$, including the 'Laura Bassi initiative', which brings together junior collaboration members interested in increasing diversity within the collaboration, and the 'Starterkit' initiative, which provides introductory tutorials on $\mathrm{LHCb}$ software and relevant physics topics.

40th International Conference on High Energy physics - ICHEP2020

July 28 - August 6, 2020

Prague, Czech Republic (virtual meeting)

\footnotetext{
${ }^{*}$ Speaker
} 


\section{Introduction}

The LHCb collaboration has recognised the importance of supporting early career scientists. Young people who are new to research and to the collaboration may have unique issues which can be addressed through a successful scheme of early career initiatives as well as support from supervisors and colleagues. These proceedings outline the problems faced by early career researchers, and the range of initiatives within the LHCb collaboration, as well as LHC-wide, used to address these issues.

\section{LHCb Early Career Gender and Diversity Office}

The LHCb Early Career Gender and Diversity (ECGD) office oversees the wellbeing and working environment of all LHCb members. The focus is on early career and gender and diversity, in particular issues related to discrimination on the grounds of gender, sexual orientation, cultural background, race and other factors. There are two ECGD officers which serve a two year term and are picked by the Collaboration Board. They are chosen to be senior members of the collaboration, such that they have the required amount of 'authority' and discretion in order to deal with potentially sensitive issues. In addition plans are underway to expand the ECGD office by two early career representatives, to be chosen by the Early Career community.

The ECGD office aims to achieve their goals in a number of ways:

- One of the ECGD officers is invited to attend Collaboration Board meetings as non-voting member, and advise the LHCb management on issues related to early career and gender and diversity.

- They are available for confidential advice on matters to do with these topics, including discrimination in the workplace and sensitive issues such as sexual harassment.

- Collate regular statistics in order to monitor the collaboration's progress on these topics, for example statistics on the number of women in certain positions within the collaboration.

- Hold regular open meetings on topics to do with ECGD; these are held four times a year at the $\mathrm{LHCb}$ week and have included topics in the past such as: being an Early Career scientist during a pandemic; Gender diversity in $\mathrm{LHCb}$; and sustainability and the environment.

\section{Early Career}

As mentioned, part of the role of the ECGD office in LHCb is to provide support for early career scientists. This is done in the ways mentioned above such as advising management on early career issues and being available for confidential advice. In addition the ECGD office holds regular events aimed at early career scientists such as those meetings at LHCb weeks as well as events in collaboration with the other LHC experiments. The ECGD has also contributed to setting up an LHC wide mentoring scheme. LHCb also awards prizes for exceptional early career scientists including the LHCb Early Career Scientists Award and the LHCb Thesis Award. These awards are given to early career scientists who have made an exceptional contribution to LHCb through 
operations, physics analysis or other contributions. These aim to give young members of the collaboration help in forwarding their career and recognition of their achievements.

It is also worth mentioning that different experiments have different definitions of an 'Early Career Scientist'. Some LHC experiments define this as age or time since finishing a PhD. This can be tricky as some people may come to the field later than others and have different life experiences. $\mathrm{LHCb}$ therefore does not have a fixed definition of what constitutes an early career scientist.

\section{Problems faced by Early career scientists}

Early career scientists who are $\mathrm{PhD}$ students, young PostDocs changing experiment or starting a career in academia can face unique problems. Some examples of these are:

- For a new $\mathrm{PhD}$ student there is a lot to learn and this can be overwhelming, $\mathrm{PhD}$ students are expected to pick up a lot of new information and skills which they may not have come across in their undergraduate degree.

- For PhD students in HEP (High Energy Physics), computing and programming is almost always a particularly important skill which is required for the $\mathrm{PhD}$. Computing is not always taught to a required level, if at all, during undergraduate degrees so students often have to teach themselves computing and software skills needed for their $\mathrm{PhD}$.

- Lack of tutorials and teaching: most PhD programs do not include any specific tutorials and teaching, it is therefore important for $\mathrm{PhD}$ students to be aware of training opportunities available to them.

- Due to the high work load in academia in general, documentation for software may exist but it can be out of date, not maintained or broken. Lack of adequate documentation can be a problem for early career scientists.

- Students may not know who to go to to ask for advice or help. LHCb has mailing lists dedicated to specific topics which can be useful; however these can have the problem of students being reluctant to use them due to fear of asking a trivial question.

- Joining a large collaboration can be intimidating and new members may struggle to meet other colleagues, network and build a community of other scientists.

\section{LHCb Early Career Initiatives}

LHCb has a number of initiatives to address the problems mentioned above; these are run by the ECGD office, and other young members of the collaboration, as well as some in collaboration with the other LHC experiments.

\section{LHCb UK Student Meetings}

LHCb student meetings are regular meetings (held once every $\sim 4$ weeks) organised by UK LHCb PhD students. They are held at CERN (pre-pandemic) and were originally started for UK 
$\mathrm{PhD}$ students based at CERN for a long-term attachment. However they have since been opened to any LHCb student or early career scientist and are now held remotely.

The meetings consist of a talk by a more senior member of the collaboration on an overview of a particular aspect of LHCb. The aim is to cover some aspect of physics analysis or detector physics which is important or unique to LHCb which students may not have been taught in detail previously. These topics include: Stripping (LHCb Event Selection), Simulation and Probability, Likelihood and Confidence, as well as some non-LHCb specific topics such as Outreach, Gender and Diversity and Science in Parliament.

\section{LHCb Startertalk}

The LHCb Startertalk is a similar initiative in the sense that it is also aimed at early career LHCb members and consists of a talk by a more senior member of the collaboration. These are held once every LHCb week, which are four times a year. The aim of the Startertalk is to create an informal environment for students so they feel able to ask questions. The format is a blackboard talk where the speaker may write down key information followed by an open discussion - this often creates a more informal environment and slower pace than with PowerPoint presentations. Previous topics for the Startertalk include: DecayTreeFitter (algorithm for kinematic reconstruction), Flavour Tagging, Particle Identification.

\section{LHCb Starterkit}

The LHCb Starterkit is a week-long workshop aimed at first year PhD students on LHCb. The workshop consists of tutorials on introductory computing and LHCb software. The Starterkit was started in 2015 by a group of early career scientists and PhD students in the LHCb collaboration in order to address some of those problems faced by first year PhD students mentioned above. The Starterkit aims to address these by providing introductory tutorials on computer programming such as python, Git and Bash and also more LHCb specific software. The aims are to give a solid starting point to newcomers in LHCb software, improve software literacy and teach good practices. The first two days of the Starterkit are usually held in collaboration with ALICE and SHiP and focus on introductory computing with lessons on Git, Bash, and Python. The second part of the workshop is an introduction to $\mathrm{LHCb}$ software.

An important part of the ethos of the Starterkit is that it was started by and is still run by early career scientists. The teachers and helpers are usually PhD students or Post Docs - PhD students who have previously participated in the Starterkit are encouraged to sign up to help or teach the following years. It is organised by two or three more experienced $\mathrm{PhD}$ students who usually change from year to year to ensure the project continues in the subsequent cohorts. It is also worth noting that teachers and helpers gain a lot from teaching material they may have learnt in previous years and they learn a lot through this experience. The material is maintained collaboratively on GitHub and is kept up to date on a yearly basis by many pull requests from students. It is important for the lessons to be kept extremely well documented and open-source so that all students can access the material even if they cannot travel to CERN for the workshop.

The Starterkit program also comprises of the Impactkit. The Impactkit is a more advanced set of LHCb specific tutorials aimed at those at the end of the first year of PhD. It is usually held over 
three days with one day consisting of a Hackathon where students are invited to work on their own projects and present to the group at the end of the day. This is an important opportunity for students to network with other students and helpers as well as practice their hacking and presentation skills.

\section{The Laura Bassi Initiative}

The Laura Bassi initiative is a junior-lead LHCb initiative bringing together people who are interested in tackling the gender diversity issue at $\mathrm{LHCb}$ via a 'bottom-up' approach. This is an informal network which aims to create an environment conducive to creative discussion and new ideas in tackling relevant issues, as well enabling people to share information about early career opportunities, provide advice and a source of support and facilitate dialogue about equal opportunities. The Laura Bassi initiative is open to anyone who feels they stand to gain from being part of this network, or who is interested in contributing to the causes it addresses. It is named after Laura Bassi who was an Italian physicist and the first woman to have a doctorate in science. Events and discussions have been organised during the LHCb collaboration weeks on topics related to gender and diversity such as Imposter Syndrome.

\section{LHC Early Career Initiatives}

In addition to the early career initiatives within $\mathrm{LHCb}$, the other LHC experiments also have a comprehensive programme of early career initiatives including those organised between all four LHC experiments. Each of the other LHC experiments has a dedicated Early Career board or committee: ATLAS has an Early Career Scientist Board which consists of seven members of a two year term; the CMS Young Scientist Committee consists of $\sim 20$ members with a chair who sits for one year; and the Junior Community in ALICE has three junior representatives with a term of two years. The key difference between these and LHCb is that the early career committees of the other experiments are separate to the gender and diversity offices whereas on LHCb they are combined.

Each of the early career boards organises a range of events and initiatives individually. There are also regular events organised by all four experiments such as the LHC Soft Skills Workshop; this includes presentations and Q\&As on certain topics relevant to young scientists such as CV and academic writing and working from home. The LHC Career Networking events consist of presentations by LHC alumni who have left academia for jobs in industry. In addition the Ice Cream events consist of informal short talks by experts for a general audience. The Career Networking Event and Ice Cream event are long-standing initiatives to which the ECGD contributes, while the series of Soft Skills Workshop is a new (2020) initiative, which the ECGD helped initiate and set up. Following feedback from the early-career community, the ECGD has joined forces with the other early-career fora to organise a joint early-career mentoring scheme with the goal of pairing mentors and mentees from different experiments.

\section{Conclusion}

The LHCb collaboration recognises the importance of supporting it's early career scientists. This is done through a wide range of early career initiatives held within the collaboration and in collaboration with the other three LHC experiments. These events and initiatives are organised 
by the experiments early career boards as well as young people on the experiment. Many of the initiatives which have been mentioned are organised exclusively by early career scientists; this highlights the importance of a bottom-up approach in tackling the unique needs of early career scientists in HEP. 